\title{
CONTINUITY OF THE VISIBILITY FUNCTION
}

\author{
Ana Forte Cunto
}

\begin{abstract}
G. Beer defined the visibility function of a set $S$ and proved its continuity in the interior of $S$. It is proved here that the visibility lunction of a planar Jordan domain $S$ is continuous precisely at the cone points of the boundary of $S$.
\end{abstract}

\section{Notations and basic definitions}

Unless otherwise stated, all the points considered here are included in the Euclidean plane $E_{2}$. The complement, interior, closure, boundary and convex hull of a set $S$ are denoted by C $S$, int $S, \mathrm{cl} S$, bd $S$ and conv $S$, respectively. The open segment joining $x$ and $y$ is denoted $(x y)$. The substitution of one or both parentheses by square ones indicates the adjunction of the corresponding endpoints. The ray issuing from $x$ and going through $y$ is denoted $R(x \rightarrow y)$, while $R(y x \rightarrow)$ is the ray issuing from $x$ and going in the opposite direction to that of $R(x \rightarrow y)$. Rays are always closed. We say that $x$ sees $y$ via $S$ if $\left[\begin{array}{ll}x & y\end{array}\right] \subset S$. The star of $x$ in $S$ is the set $\operatorname{st}(x, S)$ of all the points of $S$ that see $x$ via $S$. A star-center of $S$ is a point $x \in S$ such that $\operatorname{st}(x, S)=S$. The convex kernel of $S$ is the set ker $S$ of all the star-centers of $S . S$ is starshaped if ker $S \neq \phi$. A Jordan domain is a compact connected set of $E_{2}$ whose boundary is homeomorphic to the unit circle. The open and closed disks of center $x$ and radius $\delta$ will be denoted $U(x ; \delta)$ and $B(x ; \delta)$, respectively.

If $y \in$ bd $S$ and $x \in \operatorname{st}(y, S)$ we say that the ray $R(x \rightarrow y)$ is inward through $y$ if there exists $t \in R(x y \rightarrow)$ such that $(y t) \subset$ int $S$. Otherwise we say that $R(x \rightarrow y)$ is outward through $y$. The inner stem of $y$ with respect to $S$ is the set ins $(y, S)$ formed by $y$ and all the points of st $(y, S)$ that issue outward rays through $y$.

A point $x \in S$ is a point of local convexity if there exists $\varepsilon>0$ such that $S \cap B(x, \varepsilon)$ is convex. Otherwise, $x$ is a point of local nonconvexity. We remark that the distinction is significant only for boundary points, since every interior point is trivially of local convexity. The set of all points of local convexity of $S$ and that of all points of local nonconvexity are denoted lc $S$ and lnc $S$, respectively. It is easy to see that lc $S$ is open and lnc $S$ is closed in the relative topology of bd $S$. An obstruction zone is a connected component of lnc $S$. 
A point $x \in$ bd $S$ is a flat point if $x \in$ lc $S \cap$ lc CS. The set of all such points is denoted Ap $S . x \in$ bd $S$ is an inflection point (and the set of all inflection points is denoted ifp $S$ ) if either $x \in \operatorname{lnc} S \cap \operatorname{lnc} C S$ or $x \in \operatorname{lnc} S \cap$ lc $C S \cap$ $\operatorname{cl}(f \mathrm{fp} S)$. An arc $\Gamma \subset$ bd $S$ keeps the sense of curvature if either $\Gamma \subset$ lc $S$ or $\Gamma \subset \operatorname{lnc} S$.

If $S$ is a closed set with nonempty interior and $x \in S$, then the set of critical visibility of $x$ in $S$ is the set

$$
\operatorname{cv}(x, S)=\operatorname{int} S \cap \operatorname{bd} \operatorname{st}(x, S) .
$$

Each point of this set is a point of critical visibility of $x$ in $S$. The point $x \in S$ is clearly visible from $y$ via $S$ if there exists $\varepsilon>0$ such that $B(x, \varepsilon) \cap S \subset \operatorname{st}(y, S)$.

\section{Statement of the problem}

In [1] G. Beer defines the visibility function as the onc that assigns to each point $x$ of a fixed measurable set $S$ in the Euclidean space $\mathbb{E}_{n}$, the Lebesgue outer measure of $\operatorname{st}(x, S)$. We shall denote it $v(x)$.

In [1], [2] and [3] several theorems about the continuity of $v(x)$ in open sets, or in the interior of the sets considered, are demonstrated. The purpose of the present paper is to study the behavior of the visibility function in the boundary of a Jordan domain $S$. The study is restricted to this case in order to avoid difficulties as those presented in the examples of [2] and [3]. In this case, the theorems shown in $[\mathbf{1}]$ assurc the continuity of $v(x)$ in int $S$.

Furthermore, the boundary curve must have finitely many inflection points in the smooth case, and finitely many angular points in the nonsmooth case. This will prevent the existencc of singular points (i.e. points of accumulation of inflection points or angular points). The study of the star of a singular point scems almost unmanageable to this author. A Jordan domain without singular points will be a regular Jordan domain.

We make a local study of the star's measure in a point $x \in \mathrm{bd} S$ using the domain of good behavior $N$, that is a neighborhood of $x$ having the following characteristics:

i) its center will be $x$;

ii) $N$ includes neither infection points nor angular points of bd $S$ except possibly $x$ itself.

Clearly ii) assures that each of the two subarcs $\Gamma_{1}$ and $\Gamma_{2}$ of $N \cap$ bd $S$, having $x$ as one endpoint, keeps the sense of curvature. We generalize this local results using the fact that the stars are fans spanned by $S$. The definition of this concept is given below. 


\section{Auxiliary results}

Lemma 3.1. Let $S$ be a closed set of the plane, $\{x ; y ; z\} \subset S$ such that $[x y] \cup[y z] \subset S$. Let $T=\operatorname{conv}\{x ; y ; z\}$ have at most one point $w \in \operatorname{lnc} S$ such that $w \in(x z)$. Then $T \subset S$.

Proof: A slight variation in the proof of Corollary 2 of $[6]$ yields this lemma that is, in the same spirit of Valentine's resuit, an useful consequence of Tietze's theorem on local convexity.

Lemma 3.2. Let $S$ be a regular Jordan domain and $x_{0} \in$ bd $S$. There exists a domain of good behavior $N=B\left(x_{0}, \delta\right)$. Furthermore, $A=\left[B\left(x_{0}, \delta\right) \sim\left\{x_{0}\right\}\right] \cap$ bd $S$ consists of two connected arcs ending at $x_{0}$, such that each of them keeps the sense of curvature.

Proof: Define

$$
\begin{gathered}
K=\{z \in \text { bd } S \mid z \text { is an infection point or an angular point }\} \\
\qquad \delta=d\left(x_{0}, K \sim\left\{x_{0}\right\}\right)
\end{gathered}
$$

From the inexistence of singular points it follows that $\delta>0$. Let $\Gamma$ be the connected component of $\left[\mathrm{bd} S \cap B\left(x_{0}, \delta / 2\right)\right]$ that includes $x_{0}$. Then $\Gamma=\Gamma_{1} \cup \Gamma_{2}$ where each of these subarcs ends at $x_{0}$ and kceps the sense of curvature. Define $B_{n}=B\left(x_{0}, \delta / 2 n\right)$ and $\Gamma_{1 n}, \Gamma_{2 n}$ as the connected components of $\Gamma_{1} \cap B_{n}$ and $\Gamma_{2} \cap B_{n}$, respectively, that include $x_{0}$. Let $\Delta_{n}=\Gamma_{n} \sim\left[\Gamma_{1 n} \cup \Gamma_{2 n}\right]$.

Owing to the simplicity of bd $S$, there exists a positive integer $m$ such that $B_{m} \cap \Delta_{m}=\phi$. The ball $N=B_{m}$ satisfies the thesis.

In the sequel, the domain of good behavior with respect to $x_{0}$ will be denoted by $N$. Let $x_{0} \in$ bd $S$ and $L_{\alpha}$ be a line through $x_{0}$. The maximal segment determined by $L_{\alpha}$ in $S$ is the connected component $I_{\alpha}$ of $L_{\alpha} \cap S$ that includes $x_{0}$. The union of all those maximal segments is the fan in $x_{0}$ spanned by $S$. The angular amplitude of a fan $S(a a S)$ will be the normalized Lebesgue measure (in bd $N$ of the radial projection of $S$ from $x_{0}$ over bd $N$. $S$ will be an angular connected fan if that projection is connected in the relative topology of bd $N$.

Lemma 3.3. ins $\left(x_{0}, S\right)$ and st $\left(x_{0}, S\right)$ are fans in $x_{0}$.

Proof: Both sets are starshaped and $x_{0}$ is a star-center for each of them.

Lemma 3.4. $I=N \cap \operatorname{ins}\left(x_{0}, S\right)$ is an angular connected fan.

Proof: We consider two alternatives:

(i) Let $u \in I, v \in I$ and $x_{0}$ be not collinear with these points. There exist $u^{\prime} \in R\left(u x_{0} \rightarrow\right) \cap N$ and $v^{\prime} \in R\left(v x_{0} \rightarrow\right) \cap N$ such that $\left(x_{0} u^{\prime}\right] \cap \operatorname{int} S=\phi$ 
and $\left(x_{0} v^{\prime}\right] \cap$ int $S=\phi$. Assume that $\left(x_{0} u^{\prime}\right)$ and $\left(x_{0} v^{\prime}\right)$ are both included in $N \cap C S$. Define

$$
\begin{aligned}
& \beta=\min \left\{d\left(x_{0}, u\right) ; d\left(x_{0}, v\right) ; d\left(x_{0}, u^{\prime}\right) ; d\left(x_{0}, v^{\prime}\right)\right\} \\
& B^{\prime}=B\left(x_{0}, \beta / 2\right) \quad, \quad B_{1}^{\prime}=\mathrm{bd} B^{\prime}, \\
& u_{1} \in\left[x_{0} u\right] \cap B_{1}^{\prime} \quad, \quad v_{1} \in\left[x_{0} v\right] \cap B_{1}^{\prime} \text {, }
\end{aligned}
$$

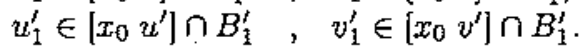

Clearly $\left(x_{0} \quad u_{1}^{\prime}\right] \subset \mathbb{C} S$, and $\left(x_{0} v_{1}^{\prime}\right] \subset \mathbb{C} S$. Furthermore, condition (i) implies that $v_{1} \notin L\left(x_{0} u_{1}^{\prime}\right)$, hence $\left[\begin{array}{ll}v_{1} & u_{1}^{\prime}\end{array}\right] \cap$ bd $S \neq \phi$. An analogous argument shows that $\left[\begin{array}{ll}u_{1} & v_{1}^{\prime}\end{array}\right] \cap$ bd $S \neq \phi$. Let $q_{1} \in\left[\begin{array}{ll}v_{1} & u_{1}^{\prime}\end{array}\right] \cap$ bd $S$ and $p_{1} \in\left[\begin{array}{ll}u_{1} & v_{1}^{\prime}\end{array}\right] \cap$ bd $S$. Hence $p_{1} \neq x_{0}$ and $q_{1} \neq x_{0}$ by condition (i), and bd $S$ cannot cross neither

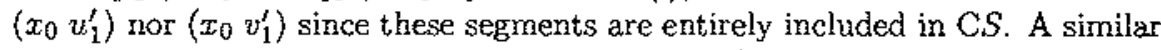
argument shows that bd $S$ cannot cross neither $\left(x_{0} u_{1}\right)$ nor $\left(x_{0} v_{1}\right)$. Since $N$ is the domain of good behaviour of $x_{0}$, only two subarcs of bd $S$ (call them $\Gamma_{1}$ and $\Gamma_{2}$ ), both having $x_{0}$ as one extreme, are included in $N$. Define two circular sectors of $B^{\prime}$ :

$$
S_{1}=\left(\begin{array}{lll}
v_{1} & x_{0} & u_{1}^{\prime}
\end{array}\right) \text { and } S_{2}=\left(\begin{array}{lll}
u_{1} & x_{0} & v_{1}^{\prime}
\end{array}\right)
$$

such that $q_{1} \in S_{1}$ and $p_{1} \in S_{2}$. It follows that

$$
\Gamma_{1} \subset S_{1}, \Gamma_{2} \subset S_{2}
$$

and

$$
\begin{aligned}
& \operatorname{int}\left(\operatorname{conv}\left\{u_{1} ; v_{1} ; x_{0}\right\}\right) \cap \text { bd } S=\phi \\
& \operatorname{int}\left(\operatorname{conv}\left\{u_{1}^{\prime} ; v_{1}^{\prime} ; x_{0}\right\}\right) \cap \text { bd } S=\phi
\end{aligned}
$$

If $z \in\left[\begin{array}{ll}u_{1} & v_{1}\end{array}\right]$, it follows from (2) and Lemma 3.1 that $\left[z x_{0}\right] \subset S$ and $z \in$ st $\left(x_{0}, S\right)$. Define now $z^{\prime} \in\left(u_{1}^{\prime} v_{1}^{\prime}\right) \cap R\left(z x_{0} \rightarrow\right)$. Using (3) and Lemrna 3.1 we obtain that $\left(x_{0} z^{\prime}\right) \subset \mathrm{C} S$, and $z \in \operatorname{ins}\left(x_{0}, S\right)$. A very similar argument halds when one or both of the segments $\left[x_{0} u_{1}^{\prime}\right],\left[\begin{array}{ll}x_{0} & v_{1}^{\prime}\end{array}\right]$ is included in bd $S$. We conclude that $I$ is convex, whence its radial projection from $x_{0}$ onto bd $N$ must be connected in the relative topology of bd $N$.

(ii) Let $u \in I, v \in I$, and $x_{0} \in(u v)$.

Since $R\left(u x_{0} \rightarrow\right)$ is outward, $x_{0} \in(u v)$ and $\left[x_{0} v\right] \subset S$, it follows that $\left[\begin{array}{ll}x_{0} & v\end{array}\right] \subset$ bd $S$. The same argument proves that $\left[x_{0} u\right] \subset$ bd $S$, and as $S$ is a Jordan domain, $I \cap B^{\prime}$ must be a half-circle. Clearly, its projection from $x_{0}$ onto bd $N$ must be connected.

Lemma 3.5. $J=N \cap \operatorname{st}\left(x_{0}, S\right)$ is an angular connected fan.

Proof: We consider three alternatives:

(I) Let $u \in J, v \in J$ and $u \in N \cap \operatorname{st}(v, S)$, while $x_{0} \notin[u v]$. Let $A=$ $\operatorname{conv}\left(\left\{u ; v ; x_{0}\right\}\right)$. Then $\operatorname{int} A \cap \mathrm{bd} S=\phi$ since any crossing of bd $S$ over 
$\left[\begin{array}{lll}x_{0} & u\end{array}\right],\left[\begin{array}{ll}x_{0} & v\end{array}\right]$ or $\left\{\begin{array}{ll}u & v\end{array}\right]$ would ruin the conditions of visibility. If $z \in\left[\begin{array}{ll}u & v\end{array}\right]_{\text {, }}$ by Lemma 3.1 it follows that $z \in J$. Hence, $J$ results convex; and its radial projection from $x_{0}$ over bd $N$ must be connected.

(2) Let $u \in J, v \in J, u \notin N \cap \operatorname{st}(v, S)$, while $x_{0} \notin[u v]$. Define

$$
\begin{array}{r}
\delta=\min \left\{d\left(u, x_{0}\right) ; d\left(v, x_{0}\right)\right\}, B^{\prime}=B\left(x_{0}, \delta / 2\right), B_{1}^{\prime}=b d B^{\prime}, \\
u^{\prime} \in\left[x_{0} u\right] \cap B_{1}^{\prime}, v^{\prime} \in\left[x_{0} v\right] \cap B_{1}^{\prime} .
\end{array}
$$

Clearly $u^{\prime} \in J, v^{\prime} \in J$, and we may assume that $u^{\prime} \notin N \cap \operatorname{st}\left(v^{\prime}, S\right)$, since otherwise we would be in the situation of part (1). Let

$$
\begin{aligned}
& z \in\left(u^{\prime} v^{\prime}\right) \cap \mathrm{C} S, p \in\left(v^{\prime} z\right) \cap \mathrm{bd} S \cap N \cap \operatorname{st}\left(v^{\prime}, S\right), \\
& q \in\left(u^{\prime} z\right) \cap \mathrm{bd} S \cap N \cap \operatorname{st}\left(u^{\prime}, S\right) .
\end{aligned}
$$

The visibility conditions assure that $p \neq x_{0}, q \neq x_{0}$ and $p \neq q$. We intend to prove that $p$ and $q$ belong to different boundary arcs separated by $x_{0}$ in bd $S \cap N$. It may happens that $p=v^{\prime}$ or $q=u^{\prime}$, but in this case one of the boundary arcs would be a segment, and since $x_{0} \notin[p q]$, these points must belong to different arcs. Let us assume that $p \neq v^{\prime}$ and $q \neq u^{\prime}$, and suppose that $p$ and $q$ belong to the same boundary arc $\Gamma_{1}$. Without loss of generality assume

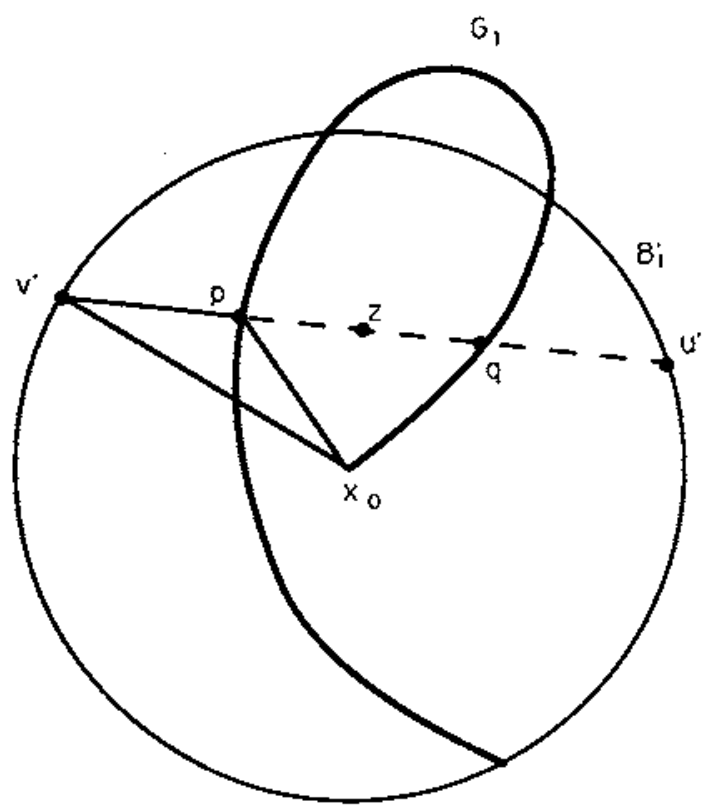

that $q \in$ arc $\left(x_{0}, p\right) \subset \Gamma_{1}$. By the definition of $p$, it is not the last point of $\Gamma_{1}$ in $B^{\prime}$. Let $A=\operatorname{conv}\left(\left\{v^{\prime} ; p_{i} x_{0}\right\}\right)$. We observe the position of $\Gamma_{1}$ with respect to $A$. If $\Gamma_{\mathrm{I}} \cap \operatorname{int} A=\phi$, either a double point or a forbidden change in the sense of curvature would appear in bd $S \cap N$. If $\Gamma_{1} \cap$ int $A \neq \phi$, it would imply a 
double point, a contradiction of the visibility conditions or a forbidden change of curvature. Hence, $p$ and $q$ must belong to different boundary arcs. Let $S_{1}=\left(v^{\prime} x_{0} u^{\prime}\right)$ the circular sector of $B^{\prime}$ that includes $z ; S_{2}=B^{\prime} \cap \mathrm{C} S_{1}$. Then bd $S \cap B^{\prime} \subset S_{1}$. Define

$$
z^{\prime} \in R\left(z x_{0} \rightarrow\right) \cap B_{1}^{\prime} .
$$

Then $z^{\prime} \in J$, and $z^{\prime} \in \operatorname{int} S_{2}$. Using part (1) we obtain that

$$
\left[u^{\prime} z^{\prime}\right] \cup\left[z^{\prime} v^{\prime}\right] \subset J,
$$

and the radial projection of this union over bd $N$ is connected.

(3) Let $u \in J, v \in J$ but $x_{0} \in[u v]$.

Let $B^{\prime}, B_{1}^{\prime}, u^{\prime}$ and $v^{\prime}$ be as in (2). Let $z \in \mathrm{C} S \cap B^{\prime}$, and take

$$
\begin{aligned}
& p \in\left[u^{\prime} z\right] \cap \operatorname{st}\left(u^{\prime}, S\right) \cap \operatorname{bd} S \\
& q \in\left[v^{\prime} z\right] \cap \operatorname{st}\left(v^{\prime}, S\right) \cap \operatorname{bd} S
\end{aligned}
$$

Since $z, u$ and $v$ are not collinear, it follows that $p \neq q$. Using that $z^{\prime}=$ $R\left(z x_{0} \rightarrow\right) \cap B_{1}^{\prime}$, and the same arguments as in (2) we obtain

$$
\left[u^{\prime} z^{\prime}\right] \cup\left[z^{\prime} v^{\prime}\right] \subset J
$$

Lemma 3.6. Let $\left\{x_{n}[n \in \mathbb{N}\}\right.$ be a sequence of points in $S$ such that $\lim x_{n}=$ $x_{0}$. Then

$$
\operatorname{ins}\left(x_{0}, S\right) \subset\left[\bigcup_{n=1}^{\infty} \bigcap_{j=n}^{\infty} \operatorname{st}\left(x_{j}, S\right)\right] \cup\left\{x_{0}\right\} \cup Q=\left[\lim _{x_{n} \rightarrow x_{0}} \operatorname{st}\left(x_{n}, S\right)\right] \cup\left\{x_{0}\right\} \cup Q,
$$

where $Q$ is included in the union of a finite number of maximal segments with respect to $x_{0}$ and has null measure.

Proof: Let $p \in \operatorname{ins}\left(x_{0}, S\right), p \neq x_{0}$. We consider two alternatives:

a) If $x_{0}$ is clearly visible from $p$, there exists a neighborhood $\mathcal{U}\left(x_{0}\right)$ such that $\mathcal{U}\left(x_{0}\right) \cap S \subset \operatorname{st}(p, S)$. Then if $x_{n} \rightarrow x_{0}, x_{n} \in \mathcal{U}\left(x_{0}\right) \cap S \forall n>n_{0}$ and $x_{n} \in \operatorname{st}(p, S) \forall n>n_{0}$. Hence

$$
p \in \bigcap_{j=n_{0}}^{\infty} \operatorname{st}\left(x_{j}, S\right) \subset \bigcup_{n=1}^{\infty} \bigcap_{j=n}^{\infty} \operatorname{st}\left(x_{j}, S\right)
$$

b) If $x_{0}$ is not clearly visible from $p$, it follows from Theorem 2.1 of [5] that $\left(p x_{0}\right) \cap \operatorname{lnc} S \neq \phi$. Let $z \in\left(p x_{0}\right) \cap \operatorname{lnc} S$. If $z$ is a smooth point of bd $S$, there exists an obstruction zone $\Gamma \subset \operatorname{lnc} S$ that includes $z$. Then conv $\Gamma$ is supported by, at most, two rays issuing from $x_{0}$. Since there are finitely many obstruction zones, and for each of them there are at most two maximal segments of critical visibility, the family of such segments is finite. If $z$ is not a smooth point of bd $S$, a similar argument, based in the definition of regular Jordan domain, assures that the family of segments of critical visibility is finite. 
Lemma 3.7. Let $F$ be an angular connected fan in $x_{0}$ and $m(F)$ its planar Lebesgue measure. Then $m(F)>0$ if and only if $a a(F)>0$.

Proof: Taking $x_{0}$ as the origin, the area of $F$ can be easily computed by a positive radial function $r(\theta)$ that depends on the argument $\theta$, whose range of variation is the projection of $F$ from $x_{0}$ over bd $N$. Let $m$ and $n$ be the endpoints of this projection. If $m, n$, and $x_{0}$ are not collinear, then let $A=$ $\operatorname{conv}\left(\left\{m ; x_{0} ; n\right\}\right)$, and $\alpha$ be the angle formed by $R\left(x_{0} \rightarrow n\right)$ and $R\left(x_{0} \rightarrow m\right)$ exterior to $A$. Then, it is clear that $a a(F)=2 \pi-\alpha$. Hence,

$$
m(F)=\int_{\alpha}^{2 \pi} \int_{0}^{r(\theta)} r d r d \theta
$$

Both implications of the thesis follow readily. Otherwise, if $m, n$ and $x_{0}$ are in the same line $L$, take $t \in F \sim L$, and $A=\operatorname{conv}\left(\left\{m, x_{0}, n, t\right\}\right)$. A very similar argument holds and (1) is valid with $\alpha=\pi$.

Lemma 3.8. Let $M$ and $L$ be two connected fans with vertex $x_{0}, M \subset L$. Then $m(M)<m(L)$ if and only if $a a(M)<a a(L)$.

Proof: The set $F=(L \sim M) \cup\left\{x_{0}\right\}$ is a fan in $x_{0}$ whose projection from $x_{0}$ over bd $N$ is not necessarily connected, but has at most two connected components. Hence $F=F_{1} \cup F_{2}$ and $L=M \cup F_{1} \cup F_{2}$. Both implications of the thesis can be obtained from equality (1) of Lcmma 3.7 and the additivity of Lebesgue measure.

Lemma 3.9. $m(\operatorname{ins}(x, S) \cap N)<m(\operatorname{st}(x, S) \cap N)$ implies that $m(\operatorname{ins}(x, S))<$ $m(\operatorname{st}(x, S))$.

Proof: Let us assume the existence of a system of polar coordinates centered at $x$ and similar to the one described in Lemma 3.7. If $\alpha$ and $\beta$ are the angular coordinates of the endpoints of the radial projection of ins $(x, S)$ over bd $N$, and $\alpha^{\prime}, \beta^{\prime}$ are the corresponding coordinates for st $(x, S)$, then aa(ins $\left.(x, S)\right)=$ $\beta-\alpha, a a(\operatorname{st}(x, S))=\beta^{\prime}-\alpha^{\prime}$. Let $\varepsilon$ be the radius of $N$. From Lemma 3.8 it follows that $\alpha^{\prime} \leq \alpha \leq \beta \leq \beta^{\prime}$. Using the notation of Lemma 3.7 it results that

$$
m(\operatorname{st}(x, S))=m(\operatorname{st}(x, S) \cap N)+\int_{\alpha^{\prime}}^{\beta^{\prime}} \int_{\varepsilon}^{r(\theta)} r d r d \theta
$$

and

$$
m(\operatorname{ins}(x, S))=m(\operatorname{ins}(x, S) \cap N)+\int_{\alpha}^{\theta} \int_{\varepsilon}^{r(\theta)} r d r d \theta
$$

and the strict incquality of the thesis follows readily. 


\section{The main theorem and its corollaries}

Theorem 4.1. Let $S$ be a regular Jordan domain and $x_{0} \in \mathrm{bd} S$. Then, the following statements are equivalent:

(i) $m\left(\operatorname{st}\left(x_{0}, S\right)\right)=m\left(\operatorname{ins}\left(x_{0}, S\right)\right)$

(ii) $v$ is continuous in $x_{0}$.

Proof: (i) $\Rightarrow$ (ii). Assume that $v$ is discontinuous in $x_{0}$. Owing to the upper semicontinuity of $v$ (Beer, [1], there must exist a sequence $\left\{x_{n}\right\}$ in $S$ such that $x_{n} \rightarrow x_{0}$ but $\lim _{n \rightarrow \infty} v\left(x_{n}\right)<v\left(x_{0}\right)$. Hence,

$$
\lim _{n \rightarrow \infty} m\left(\operatorname{st}\left(x_{n}, S\right)\right)<m\left(\operatorname{st}\left(x_{0}, S\right)\right)
$$

From Lemma 3.6 it follows that

$$
\operatorname{ins}\left(x_{0}, S\right) \subset\left[\bigcup_{n=1}^{\infty} \bigcap_{j=n}^{\infty} \operatorname{st}\left(x_{j}, S\right)\right] \bigcup\left\{x_{0}\right\} \bigcup Q
$$

where $Q$ has null measure. From (2) it follows that

$$
\begin{aligned}
m\left(\operatorname{ins}\left(x_{0}, S\right)\right) \leq m\left[\bigcup_{n=1}^{\infty} \bigcap_{j=n}^{\infty} \operatorname{st}\left(x_{j}, S\right)\right]+m\left(\left\{x_{0}\right\}\right)+m(Q)= \\
\qquad \lim _{n \rightarrow \infty} m\left[\bigcap_{j=n}^{\infty} \operatorname{st}\left(x_{j}, S\right)\right] \leq \lim _{n \rightarrow \infty} m\left(\operatorname{st}\left(x_{n}, S\right)\right) .
\end{aligned}
$$

From this inequality and (1) we obtain a contradiction of (i).

(ii) $\Rightarrow$ (i). Assume that $m\left(\operatorname{st}\left(x_{0}, S\right)\right) \neq m\left(\operatorname{ins}\left(x_{0}, S\right)\right)$. It is clear that $m\left(\operatorname{st}\left(x_{0}, S\right)\right)-m\left(\operatorname{ins}\left(x_{0}, S\right)\right)>0$, and that

$$
D=\left[\operatorname{st}\left(x_{0}, S\right) \sim \operatorname{ins}\left(x_{0}, S\right)\right]
$$

is a fan at $x_{0}$ having positive measure. From Lemma 3.8 it follows that $a a\left(\operatorname{st}\left(x_{0}, S\right)\right)>a a\left(\operatorname{ins}\left(x_{0}, S\right)\right)$, whereas from Lemmas 3.3 and 3.4 we know that both $N \cap$ ins $\left(x_{0}, S\right)$ and $N \cap$ st $\left(x_{0}, S\right)$ are angularly connected. Then, the projection of $D$ from $x_{0}$ over $N_{1}$ has at most two connected components, and at least one of them with positive measure. Hence, it is clear that $m\left(\operatorname{st}\left(x_{0}, S\right) \cap N\right)>$ $m\left(\operatorname{ins}\left(x_{0}, S\right) \cap N\right)$, and that there exists a set $A \subset D \cap N$ such that int $A \neq \phi$. Select $t \in \operatorname{int} A$ and $w \in \operatorname{ins}\left(x_{0}, S\right)$, and call $L\left(w, x_{0}\right)$ the line through $w$ and $x_{0}$ and $H^{+}, H^{-}$the two open semiplanes in which this line divides $\mathbb{E}_{2}$. Since $t \notin L\left(w, x_{0}\right)$ we can assume that $t \in H^{+}$. There exists $\varepsilon>0$ such that $B(t, \varepsilon) \subset A \subset D \cap N$. Since $R\left(t \rightarrow x_{0}\right)$ is inward, there exist a point $t^{\prime} \in R\left(t x_{0} \rightarrow\right) \cap H^{-} \cap S \cap N$ with $\left(t^{\prime} x_{0}\right) \subset$ int $S$. Clearly $t^{\prime} \notin \operatorname{ins}\left(x_{0}, S\right)$ and 
$t$ is not clearly visible form $t^{\prime}$. Let $t_{n}=\frac{1}{n} t^{\prime}+\frac{n-1}{n} x_{0}$. Hence $\forall n t_{n} \in\left(t^{\prime} x_{0}\right)$ and $t_{n} \rightarrow x_{0}$. Furthermore, each of the $t_{n}$ has the same visibility restrictions as $t^{\prime}$ with respect to $t$. Let $L\left(t, x_{0}\right)$ be the line through $t$ and $x_{0}$, and $U$ be the connected subset of $S$ limited by $L\left(t, x_{0}\right)$ and not visible from $t^{\prime}$ via $S$. Furthermore, the points of $U$ are not visible via $S$ from each of the $t_{\pi}$. Call $\tilde{S}=S \sim U$ and let $\tilde{v}$ be the visibility function of $\tilde{S}$. Clearly it holds

$$
\forall n v\left(t_{n}\right)=v\left(t_{n}\right), \tilde{v}\left(x_{0}\right)<v\left(x_{0}\right)
$$

and from the upper semicontinuity of $v$ we obtain

$$
\lim _{n \rightarrow \infty} v\left(t_{n}\right)=\lim _{n \rightarrow \infty} \tilde{v}\left(t_{n}\right) \leq \tilde{v}\left(x_{0}\right)<v\left(x_{0}\right)
$$

that contradicts (ii).

We say that $x \in$ bd $S$ is a cone point if there exists a line $L$ through $x$ such that $\operatorname{st}(x, S)$ is included in one of the two closed scmiplanes determined by $L$.

Lemma 4.2. If $S$ is a Jordan domain and $x$ is a boundary point of $S$, there exists a line $L$ through $x$ that leaves $\operatorname{ins}(x, S)$ at one side of it.

Proof: As lemma 3.4 states, ins $(x, S) \cap N$ is an angular connected fan. If such a line does not exist, there must be a line through $x$ that intersects the radial projection from $x_{0}$ of ins $(x, S)$ on bd $N$ in two points $\left\{t_{1} ; t_{2}\right\}$, where at least one of them (say $t_{1}$ ) is not an endpoint of that projection. Hence there should exist $t_{0} \in\left(x_{0} t_{1}\right)$ such that $\left(x_{0} t_{0}\right)$ be included into int $S$. But this should contradict the fact that $t_{2} \in$ ins $(x, S)$.

Theorem 4.3. The points of continuity of the visibility function of $S$ on the boundary of $S$ are precisely the cone points of that boundary.

Proof: Let $x$ be a cone point of bd $S$, and $L$ a line through $x$ that divides $\mathbb{E}_{2}$ into two open semiplanes $H^{+}$and $H^{-}$such that $\operatorname{st}(x, S) \subset \operatorname{cl}\left(H^{+}\right)$. Take $u \in \operatorname{si}(x, S) \sim L$, and let $u^{\prime} \in R(u x \rightarrow)$ such that $\left(x u^{\prime}\right] \subset H^{-}$. Since $u^{\prime} \notin \mathrm{st}(x, S)$, we have two alternatives:

(a) $\left(x u^{\prime}\right) \cap S=\phi$.

(b) $\left(x u^{\prime}\right) \cap S \neq \phi$ and $\left(x u^{\prime}\right) \cap C S \neq \phi$.

Each of this alternatives produces easily a point $t \in\left(x u^{\prime}\right]$ such that $(x t) \cap$ int $S=\phi$, whencc $u \in \operatorname{ins}(x, S)$. We have shown that

$$
\operatorname{st}(x, S) \sim \operatorname{ins}(x, S) \subset L \cap S
$$

and the last set has null measure. Hence $x$ satisfies hypothesis (i) of Theorem 4.1 and $v$ is continuous at $x$.

Conversely, assume that $v$ is continuous at $x$. From Lemma 4.2 there exists a line $L$ that produces a closed semiplane $H^{+}$including ins $(x, S)$. Repeating arguments used in the second part of Theorem 4.1 we obtain that the set $D=\operatorname{st}(x, S) \sim \operatorname{ins}(x, S)$ is a fan having one or two connected components and has null measure. It follows that $D \subset L$, whence st $(x, S) \subset H^{+}$and $x$ is a cone point. 
Theorem 4.4. A smooth point of bd $S$ is a cone point.

Proof: For flat points, the theorem is almost trivial. In the case of inflection. and concave points (i.e. points of lnc $S \cap \mathrm{Ic} C S$ ), the thesis follows from Lemma 4.2 and Theorems 2.4 and 2.5, respectively, from [4]. If $x$ is a convex point (that is $x \in \operatorname{lc} S \cap \operatorname{lnc} C S$ ), let $\Gamma$ be an arc of bd $S$ such that $x \in \Gamma$ and $\Gamma \subset \operatorname{lc} S \cap \operatorname{lnc} \mathrm{C} S$. Let $K=$ conv $\Gamma$ and $T(x)$ be the tangent line to $K$ through $x$. Call $H^{+}$and $H^{-}$the two open halfplanes determined by $T(x)$, where $K \subset$ cl $H^{+}$. Hence, $w \in H^{-}$implies $[w x] \sqcap \subset C S \neq \phi$, since $T(x)$ is supporting. Then $\operatorname{st}(x, S) \subset \operatorname{cl} H^{+}$and $x$ is a cone point.

Corollary 4.5. The visibility function is continuous at smooth points.

Proof: Inmediate from 4.3 and 4.4.

\section{References}

1. Beer, G, The index of convexity and the visibility function, Pacific $J$. 44 (1973), 59-67.

2. BEER, G., The continuity of the visibility function on a starshaped set, Canadian J. 24 (1972), 989-992.

3. BEeR, G., Continuity properties of the visibility function, Michigan Math. J. 20 (1973), 297-302.

4. Forte Cunto, A. AND Toranzos, F, Visibility inside a smooth Jordan domain, submitted for publication.

5. Toranzos, F.A., Critical visibility and outward rays, J. of Geometry $\mathbf{3 3}$ (1988), 155-167.

6. Valentine, F.A., Local convexity and $L_{\pi}$ sets, Proceedings of the Amer. Math. Soc. 16 (1965), 1305-1310.

\footnotetext{
Departamento de Matemática

Universidad de Buenos Aires

Pabellón 1 - Ciudad Universitaria

1428 Buenos Aires

ARGENTINA
}

Primeta versió rebuda el 17 d'Octubre de 1989 , darrera versió rebuda el 22 de Març de 1990 